\title{
THE SOURCES OF THE HARD X-RAY BACKGROUND
}

\author{
GIANCARLO SETTI AND ANDREA COMASTRI \\ Dipartimento di Astronomia, Università di Bologna \\ Osservatorio Astronomico di Bologna \\ Istituto di Radioastronomia del CNR \\ Via P. Gobetti, 101 - 40129 Bologna - Italy
}

\section{Introduction}

The hard component ( $3 \mathrm{keV}-\sim \mathrm{MeV}$ ) of the X-ray background (XRB) comprises the largest portion, $\sim 90 \%$, of the overall XRB intensity. The observed isotropy (the entire Galaxy is transparent above $3 \mathrm{keV}$ ) provides a prima facie evidence of its prevailing extragalactic nature. A large fraction ( $~ 75 \%$ ) of the energy flux falls in the $3-100 \mathrm{keV}$ band, the corresponding energy density being $\simeq 5 \times 10^{-5} \mathrm{eV} \mathrm{cm}^{-3}$, of which $50 \%$ is confined to the narrower 3-20 keV band. Although the energy flux carried by the XRB is relatively small compared to other extragalactic backgrounds, it was soon realized that it cannot be accounted for in terms of sources and processes confined to the present epoch. An analysis of the combined observed spectra (Gruber 1992) concludes that, while a thermal bremsstrahlung with an efolding energy $=41.13 \mathrm{keV}$ accurately fits the data up to $60 \mathrm{keV}$, above this energy the sum of two power laws is required with normalizations such that at $60 \mathrm{keV}$ the spectral index is $\sim 1.6$, gradually flattening to $\sim 0.7$ at $\mathrm{MeV}$ energies. It should also be noted that below $10 \mathrm{keV}$ the XRB energy spectrum is well represented by a power law of index $\alpha=0.4\left(I \propto E^{-\alpha}\right)$.

The $40 \mathrm{keV}$ thermal bremsstrahlung has been cosidered by some as evidence of a hot $\left(>10^{8} \mathrm{~K}\right)$ diffuse intergalactic gas (IGG). Subsequent investigations (Taylor \& Wright 1989 and ref. therein) have shown that a viable model, in the framework of the big-bang cosmology, can only be obtained by assuming that the IGG has been suddenly reheated at a redshift $z=3-5$ to a temperature $\mathrm{T} \sim\left(1+z_{*}\right) 40 \mathrm{keV}$ and its density $\Omega_{B}>0.2$, even larger than that allowed by the primordial nucleosynthesis. The ensuing Compton distortion of the CMB is by far inconsistent with COBE 
FIRAS results: the contribution of a hot diffuse IGG to the XRB cannot be more than $10^{-4}$ (Wright et al. 1994). Strong clumping can help in circumventing these difficulties. Nevertheless, consistency with the upper limits on the temperature fluctuations of the CMB requires a large number of unrealistically small size clumps (< few tens kpc; Barcons \& Fabian 1988). Indeed, several authors (e.g., Giacconi \& Zamorani 1987) have pointed out that the close resemblance of the XRB spectrum to that of a $40 \mathrm{keV}$ thermal bremsstrahlung provides in itself a strong argument against any interpretation involving a hot IGG as the main contributor to the XRB intensity. This is because any reasonable subtraction of the integrated contributions from known classes of extragalactic X-ray sources would destroy the almost perfect thermal shape which, therefore, must be considered as accidental.

Presently, there are no other models which may satisfactorely explain the hard XRB in terms of diffuse processes taking place in the intergalactic medium. As a consequence, the only alternative possibility is that of the summed contribution from extragalactic sources. Among these the AGN, in particular Seyfert (Sy) galaxies and quasars, are known for some time (Setti \& Woltjer 1973) to be the most likely candidates. However, unlike the situation in the soft X-rays, only a small fraction of the hard XRB has been actually resolved into sources: the HEAO- 1 A2 all-sky survey has resolved $\sim 1 \%$ of the $2-10 \mathrm{keV} \mathrm{XRB}$ down to a flux limit $\simeq 3 \times 10^{-11} \mathrm{erg} \mathrm{cm}^{-2}$ $\mathrm{s}^{-1}$ and determined a Euclidean Log $\mathrm{N}-$ Log $\mathrm{S}$ (Piccinotti et al. 1982). At these bright fluxes AGNs and clusters of galaxies are the two dominant classes of sources. $\mathrm{P}(\mathrm{D})$ fitting techniques applied to the fluctuations in the XRB measured by GINGA (Warwick \& Stewart 1989; Hayashida 1990) and to those of the HEAO-1 A2 experiment (Shafer 1983) are consistent with a Euclidean slope of the source number counts extrapolated from the Piccinotti et al. $\log \mathrm{N}-\log \mathrm{S}$ down to a flux $\sim 10^{-13} \mathrm{erg} \mathrm{cm}^{-2} \mathrm{~s}^{-1}$, at which flux about $15 \%$ of the $2-10 \mathrm{keV}$ XRB is resolved.

The main difficulty with the idea that the AGNs could supply the bulk of the XRB has been that their hard X-ray spectra $(2-20 \mathrm{keV})$ are well represented by a power law of mean energy spectral index $\langle\alpha\rangle=0.7$ (Turner \& Pounds 1989, and ref. therein), much steeper than that of the XRB. Recent developments have permitted to overcome this ostacle.

\section{Solving the spectral problem}

One of the achievements of the GINGA mission has been the discovery that the X-ray spectra of a large fraction of a sample of Sy 1 galaxies flatten beyond $\sim 10 \mathrm{keV}$, the mean spectral index in the $10-18 \mathrm{keV}$ interval being $\langle\alpha\rangle=0.28$ with $\sigma=0.15$ (Nandra \& Pounds 1994). This fact has been interpreted either as photoelectric absorption (partial coverage) of an 
underlying power law continuum source or as a hump produced by reprocessed X-rays (reflection) from thick cold $\left(<10^{6} \mathrm{~K}\right)$ matter surrounding the central source, possibly in an accretion disk around a massive black hole (Matsuoka et al. 1990; Pounds et al. 1990). The Compton reflection model is generally thought to be more representative of the physical conditions prevailing in the AGNs.

As conjectured by Schwartz \& Tucker (1988), a flattening of the average AGN spectrum above $\sim 10 \mathrm{keV}$ may provide a solution to the AGN vs. XRB spectral problem, simply because the redshifted contributions from the flat portion of the source spectra would dominate the $3-30 \mathrm{keV}$ XRB spectral shape, if the AGN volume emissivity adequately increases as a function of look-back time out to a redshift cut-off $z=3$. The rather sharp decline of the XRB spectrum above $30 \mathrm{keV}$ could be modeled by introducing a high energy cut-off at $\sim 100 \mathrm{keV}$. Following this recipe Morisawa et al. (1990), by adopting a partial coverage absorption model on a power law continuum ( $\alpha=0.7)$, obtained a good fit of the 3-100 keV XRB spectrum with parameters typical of the Sy 1 spectra measured by GINGA, assumed to be exponentially cut-off at a computed energy of $\sim 115 \mathrm{keV}$, and with a reasonable cosmological evolution of the local AGN X-ray luminosity function (XLF) from the HEAO-1 bright sample of Piccinotti et al. (1982).

Fabian et al. (1990) emphasized the physical nature of the relatively sharp break of the XRB spectrum at $\sim 30 \mathrm{keV}$ and noted that this feature can be adequately reproduced by the redshifted Compton reflection spectrum of sources at $z \sim 2$, thus avoiding the need of introducing artificial cut-offs. It was found that the fit of the XRB spectrum from several $\mathrm{keV}$ to $\sim 1 \mathrm{MeV}$ requires a new class of strong $\left(\leq 10^{45} \mathrm{erg} \mathrm{s}^{-1}\right)$ sources with more than $90 \%$ of the emitted flux in the reflected component, and a strong cosmological evolution such that the comoving volume emissivity of the sources increases as $(1+z)^{4}$ out to a redshift $z \sim 5$. 'Reflection' models of this type have been further investigated by Rogers \& Field (1991) and Terasawa (1991) with similar conclusions on the strength of the reflected component. While the new class of AGNs postulated by Fabian et al. could not have been hitherto detected, Terasawa's model assumes the local XLF of Piccinotti et al. and, therefore, the conclusion that $\sim 80 \%$ of the source flux is channeled in the reflected component exceeds the average value found in the Sy 1 spectra. A general difficulty with these models is that the predicted source counts and/or spectra in the soft X-ray band are inconsistent with those of the EMSS and ROSAT surveys (Setti 1992). It has also been pointed out (Zdziarski et al. 1993a) that these models do not adequately fit the position and the width of the peak of the XRB intensity.

Recently, another 'reflection' model has been proposed by Zdziarski et al. (1993b). Here the typical Sy 1 spectrum is made by Compton reflection 
of $\sim 50 \%$ of a primary X-ray flux produced by thermal Comptonization of seed photons in a hot plasma cloud with a Thompson depth of a few and a temperature $\mathrm{kT}=40 \mathrm{keV}$, which means that the spectrum is exponentially cut-off at $\sim 100 \mathrm{keV}$. By adopting the cosmological evolution derived by Boyle et al. (1993) from the analysis of the combined AGN sample from the EMSS and ROSAT surveys, it is found that a very good fit of the 3-100 keV XRB spectrum can be obtained with the evolution extended up to $z=4$ and with a local volume emissivity about 2 times that of Boyle et al. (1993). No details are given about the expected source counts. However, the adopted source spectral shape, in combination with the high redshifts involved in the model, is likely to produce too many sources with too flat spectra in the soft $\mathrm{X}$-rays.

In general, it appears that the 'reflection' models are rather inefficient in producing the XRB, unless a completely new class of AGNs is postulated.

Alternative models for the synthesis of the XRB assume the existence of a large population of heavily absorbed AGNs. In particular, Setti \& Woltjer (1989) have discussed a model based on the X-ray properties of AGN unified schemes, introduced by Antonucci \& Miller (1985) for the Seyfert galaxies, in which the central source can be hidden (depending on the viewing angle) by a thick torus of surrounding absorbing matter. Radioloud quasars and strong radio galaxies can be similarly unified, the quasar phenomenon showing up whenever the source axis is aimed toward us within a specified angle (Barthel 1989), while the existence of a hidden population of radio-quiet quasars is still uncertain, although the luminous IR galaxies have been proposed as likely candidates. Accordingly, it has been shown that the X-rays can be absorbed up to $>20 \mathrm{keV}$ whenever the lines of sight are intercepted by the tori, while above the absorption cut-offs the $\mathrm{X}$-ray properties should be the same as those of the 'unabsorbed' AGNs, unless the X-ray emission itself is largely beamed. X-ray observations of Sy 2 galaxies and strong radio galaxies support this hypothesis (Awaki et al. 1991; Allen \& Fabian 1992). By adopting source spectra with the canonical $2-10 \mathrm{keV}$ slope $(\alpha=0.7)$, very simple fractional distributions of the absorption cut-offs and an internally consistent cosmic evolution of the AGN XLF out to $z=3$, Setti \& Woltjer demonstrated that the $3-30$ keV XRB spectral intensity can be accurately reproduced with a number of 'absorbed' AGNs about equal to that of the 'unabsorbed' ones. Very good fits of the XRB have been obtained by more detailed investigations, thus confirming the basic validity of this scenario (Madau et al. 1993; Matt and Fabian 1994).

We have briefly reviewed a variety of models meant to explain the hard $\mathrm{XRB}$ as a superposition of the emission from AGNs. We have also underlined that it is critically important to check these models against other 
observational constraints, in particular the predicted source counts and spectra in the soft X-rays. One important feature of Setti \& Woltjer's proposal is that the 'absorbed' AGNs, essential to explain the XRB above several $\mathrm{keV}$, may not show up significantly in the soft $\mathrm{X}$-rays, so that these two spectral regions are to some extent indepedent. Therefore, the next step is the construction of a model consistent with the main statistical properties of AGN X-ray samples. An attempt in this direction by Comastri et al. (1994) will be briefly described in the following section (see also Madau et al. 1994).

\section{A baseline model of the XRB from AGNs}

The basic assumptions of the model, aimed at reproducing the XRB spectrum in the $3-100 \mathrm{keV}$ interval, can be summarized as follows:

a) A typical, primary AGN continuum spectrum with spectral indices $\alpha$ $=1.3$ below $1.5 \mathrm{keV}$ and $\alpha=0.9$ above this energy, exponentially cut-off at an e-folding energy of $\simeq 320 \mathrm{keV}$. A Compton reflection component ( $50 \%$ of the primary flux) has been added to the continuum of the low luminosity AGNs ( $<7 \times 10^{43} \mathrm{erg} \mathrm{s}^{-1}$ in the $0.3-3.5 \mathrm{keV}$ interval). This is consistent with the broad spectral characteristics observed in Sy 1 and quasars. b) The AGNs are surrounded by tori of absorbing material (solar composition) with $45^{\circ}$ half opening angle, such that hydrogen column densities $N_{H}$ of up to $10^{25} \mathrm{~cm}^{-2}$ can be intercepted, depending on the source characteristcs and orientation with respect to the line of sight to the central source. c) The adopted $(z=0)$ XLF is that determined by Boyle et al. (1993) in the $0.3-3.5 \mathrm{keV}$ band, evolved in luminosity by $(1+z)^{2.6}$ up to $z=2.25$, thereafter constant and cut-off at $z=4$. It covers a wide luminosity range from $10^{42}$ to $10^{47} \mathrm{erg} \mathrm{s}^{-1}$.

The number distribution of 'absorbed' sources as a function of $N_{H}$ (> $10^{21} \mathrm{~cm}^{-2}$ ) has been parameterized by dividing the $N_{H}$ range into four equal intervals on a $\log$ scale and by assigning to the absorbed objects the mean $N_{H}$ value of the corresponding interval. It is found that the observational constraints are met by a number distribution of 'absorbed' AGNs, normalized to the 'unabsorbed' ones, given by $0.35,1.10,2.30,1.65$, in order of increasing $N_{H}$.

Specifically, the following constraints have been identified and checked for consistency: 1) The XRB from several $\mathrm{keV}$ to about $100 \mathrm{keV}$ is well approximated (Fig. 1) to better than $5 \%$ accuracy with respect to the best fit curve of Gruber (1992). Below $\sim 5 \mathrm{keV}$ the fit underestimates the background intensity by $5-10 \%$ to allow for possible contributions from other classes of objects. 2) The source counts in the 2-10 keV band (Fig. 2) are consistent with the AGN surface density of the bright sample from the 


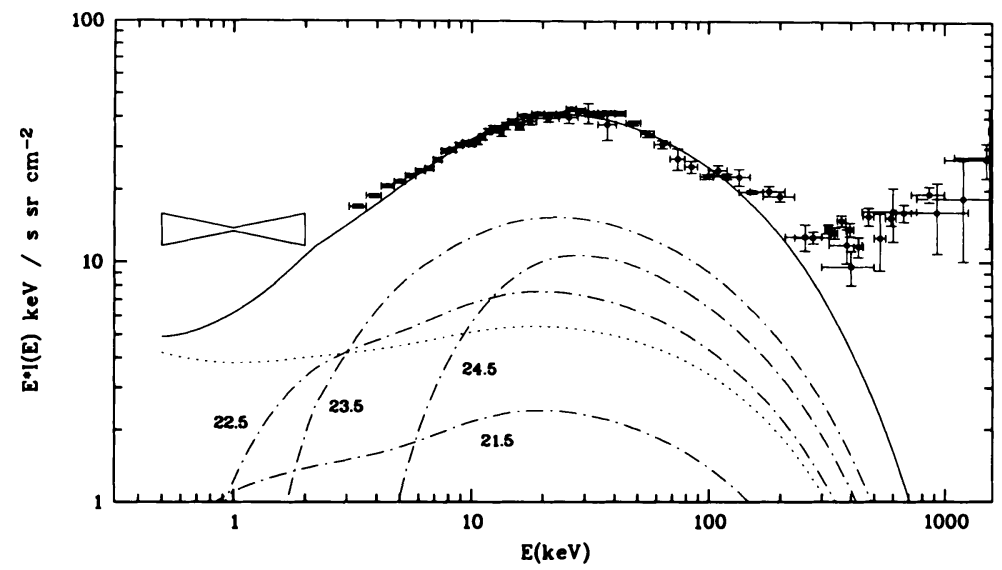

Figure 1. The XRB spectrum: $0.5-2.0 \mathrm{keV}$ data from ROSAT (Hasinger et al. 1993) and $>3 \mathrm{keV}$ from a compilation of Gruber (1992). Represented: the fit of the AGN baseline model (solid line), the contributions from unabsorbed sources (dotted line) and absorbed ones (dot-dashed lines; labels $=\log N_{H}$ ).

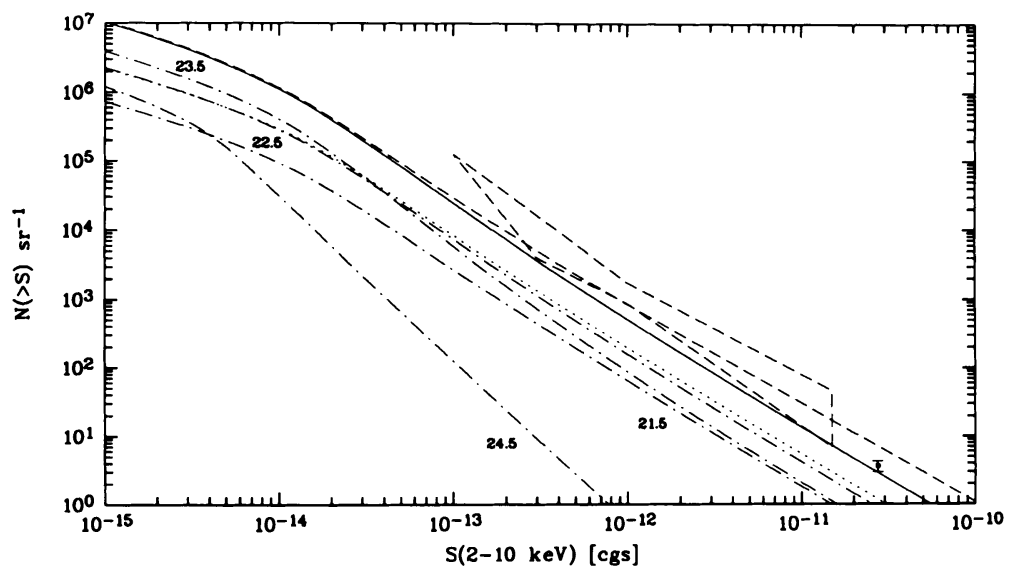

Figure 2. Baseline model predicted counts, AGN (solid) and AGN + galaxy clusters (upper dashed) compared with AGN surface density (•;Piccinotti et al. 1982) and GINGA $P(D)$ counts (dashed area). Represented: counts from unabsorbed sources (dotted) and from absorbed ones (dot-dashed; labels $=\log N_{H}$ ).

HEAO-1 A2 all-sky survey and with the constraints from the fluctuation analysis of GINGA fields. The local X-ray volume emissivity $\left(3.8 \times 10^{38} \mathrm{erg}\right.$ $\mathrm{s}^{-1} \mathrm{Mpc}^{-3}$ ) is fully consistent with that obtained by Miyaji et al. (1994) from a cross-correlation of the HEA0-1 A2 maps with the IRAS galaxies. 3) The redshift and absorption $\left(N_{H}\right)$ distributions of bright AGNs are in good agreement with those of the Piccinotti et al. sample. 4) The predicted source 


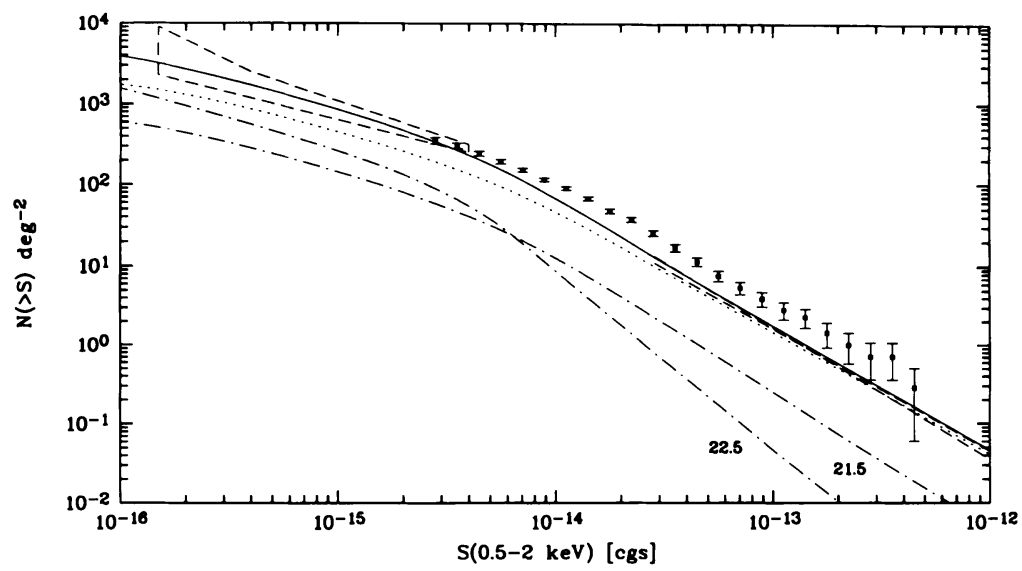

Figure 3. AGN baseline model predicted counts (solid) compared with EMSS AGN counts (dashed; Della Ceca et al. 1992), with ROSAT total counts and P(D) extension (dashed area; Hasinger et al. 1993). Represented: counts from unabsorbed sources (dotted line) and from absorbed ones (dot-dashed; labels $=\log N_{H}$ ).

counts in the soft X-rays (Fig. 3) are in good agreement with the EMSS Log N - Log S (Della Ceca et al. 1992) and with the ROSAT deep survey counts (Hasinger et al. 1993). An extrapolation of the predicted counts down to a zero flux accounts for $\sim 74 \%$ of the $1-2 \mathrm{keV}$ ROSAT XRB. Moreover, and most important, the source spectra at different flux levels have mean slopes in agreement with those found in the EMSS and ROSAT surveys. 5) The redshift distributions of the EMSS AGN sample and of the AGNs so far identified in five deep ROSAT fields are well reproduced by the model.

The ratios between absorbed and unabsorbed objects of the baseline model cannot be immediately associated with the Sy 2 / Sy 1 ratio. This is because objects with intrinsic absorptions corresponding to $N_{H}$ values of up to several times $10^{22} \mathrm{~cm}^{-2}$ have been frequently classified as Sy 1 galaxies and, viceversa, Sy 2 galaxies sometimes have $N_{H}<10^{22} \mathrm{~cm}^{-2}$. If one takes column densities in the range $10^{22}-10^{22.5} \mathrm{~cm}^{-2}$ as the dividing line between the two types, then the model predicts a ratio Sy 2 / Sy 1 $=2.4-3.7$, in good agreement with that found by Huchra \& Burg (1992) from a complete sample of optically selected Sy galaxies.

\section{Conclusions}

The main conclusion to be drawn is that it is possible, in the framework of the simplest version of the AGN unified schemes, to construct a baseline model which accurately explain the $5-100 \mathrm{keV}$ XRB spectral intensity and is consistent with the available data both in the soft and hard X-rays. The 
'absorbed' AGNs are an essential feature of the model. The contribution of the AGNs to the soft XRB measured by ROSAT is only about $75 \%$. The spectrum above $100 \mathrm{keV}$, not discussed here, could be due to a sub-class of AGNs with flat X-ray spectra up to high energies, such as is the case of the flat spectrum quasars and BL Lacs detected by CGRO/EGRET possibly contributing the $\gamma$-ray background above $100 \mathrm{MeV}$ (Setti \& Woltjer 1994). The ongoing observations with the Japanese satellite ASCA should be able to further check the validity of this model.

\section{References}

Allen S.W. \& Fabian A.C. 1992, Mon. Not. R. astr. Soc., 258, 29P

Antonucci R.R.J. \& Miller J.S. 1985, Astrophys. J., 287, 621

Awaki H., Koyama K., Inoue H., Halpern J.P. 1991, Pub. Astr. Soc. Japan, 43, 195

Barcons, X. \& Fabian, A.C. 1988, Mon. Not. R. astr. Soc., 230, 189

Barthel P.D. 1989, Astrophys. J., 336, 606

Boyle B.J., et al. 1993, Mon. Not. R. astr. Soc., 260, 49

Comastri A., Setti G., Zamorani G., Hasinger G. 1994, Astron. Astrophys., in press

Della Ceca R., Maccacaro T., et al. 1992, Astrophys. J., 389, 491

Fabian A.C., George I.M., Miyoshi S., Rees M.J. 1990, Mon.Not.R.astr. Soc., 242, 14P

Giacconi R. \& Zamorani G. 1987, Astrophys. J., 313, 20

Gruber D.E. 1992, in The $X$-ray background, eds. X. Barcons and A.C. Fabian (Cambridge Univ. Press, Cambridge), p.44

Hasinger G., et al. 1993, Astron. Astrophys., 275, 1

Hayashida K. 1990, Ph.D. Thesis, Tokyo University, ISAS RN 466

Huchra J., \& Burg R. 1992, Astrophys. J., 393, 90

Madau P., Ghisellini G., Fabian A.C. 1993, Astrophys. J., 410, L7

Madau P., Ghisellini G., Fabian A.C. 1994, Mon. Not. R. astr. Soc., 270, L17

Matsuoka M., Piro L., Yamauchi M., Murakami T. 1990, Astrophys. J., 361, 440

Matt G. \& Fabian A.C. 1994, Mon. Not. R. astr. Soc., 267, 187

Miyaji T., Lahav O., Jahoda K., Boldt E. 1994, Astrophys. J., in press

Morisawa K., Matsuoka M., Takahara F., Piro L. 1990, Astron. Astrophys., 236, 299

Nandra K. \& Pounds K.A. 1994, Mon. Not. R. astr. Soc., 268, 405

Piccinotti G., et al. 1982, Astrophys. J., 253, 485

Pounds K.A., et al. 1990, Nature, 344, 132

Rogers R.D. \& Field G.B. 1991, Astrophys. J., 378, 117

Schwartz D.A. \& Tucker W.H. 1988, Astrophys. J., 332, 157

Setti G. \& Woltjer L. 1973, in IAU Symposium No. 55, X-and Gamma-Ray Astronomy, eds. H. Bradt and R. Giacconi (Reidel, Dordrecht), p.208

Setti G. 1992, in The X-ray background, eds. X. Barcons and A.C. Fabian (Cambridge Univ. Press, Cambridge), p.187

Setti G. \& Woltjer L. 1989, Astron. Astrophys., 224, L21

Setti G. \& Woltjer L. 1994, Astrophys. J. Suppl., 82, 629

Shafer R.A. 1983, Ph.D. Thesis, University of Maryland, NASA Tech.Mem. 85029

Taylor G.B. \& Wright E.L. 1989, Astrophys. J., 339, 619

Terasawa N. 1991, Astrophys. J., 378, L11

Turner T.J. \& Pounds K.A. 1989, Mon. Not. R. astr. Soc., 240, 833

Warwick R.S. \& Stewart G.C. 1989, in $23^{\text {rd }}$ ESLAB Symposium, in Two-Topics in X-Ray Astronomy, ESA SP-296, Vol.2, p.727

Wright E.L., et al. 1994, Astrophys. J., 420, 450

Zdziarski A.A., Zycki P.T., Svensson R., Boldt E. 1993a, Astrophys. J., 405, 125

Zdziarski A.A., Zycki P.T., Krolik J.H. 1993b, Astrophys. J., 414, L81 